\title{
Combined Wave and Current Flow Close to a Rippled Bed
}

\author{
Janaka J. Wijetunge \\ University of Peradeniya
}

\begin{abstract}
This päper describes an experimental study carried out in a laboratory water tunnel to examine the velocity distribution in combined wave and current flow close to a rippled bed. The fluid velocities, both the horizontal and the vertical components, were measured simultaneously with a laser Doppler anemometer. The measurements indicate that the addition of a steady current causes a significant effect on the near-bed ensemble velocity field of the wave motion, owing primarily to the asymmetry of the lee-vortices that are formed during each half cycle of oscillation. On the other hand, the effect of adding a wave on the time-mean velocity field of a steady current is also significant in that the current experiences an enhanced roughness due to the additional turbulence produced by the wave motion.
\end{abstract}

Keywords: Waves, Currents, Rippled Bed, Boundary Layer, Velocities, Shear Stresses

\section{Introduction}

The fluid dynamics in coastal regions are usually due to both surface waves and currents. In the inner continental shelf, the fluid motions associated with the waves and currents generally extend to the sea bottom. Accordingly, the way in which waves and currents interact, particularly close to the seabed, controls and affects many phenomena of practical importance in coastal engineering. For example, the near-bed flow is primarily responsible for determining the sediment transport rate, and consequently, the erosion and siltation trends in near-shore areas. Bottom boundary layer flow dynamics also enter in fluid force calculations for seabed pipelines and other near-bed structures. Moreover, the attenuation of waves approaching the coast and the transport of pollutants are also influenced by what goes on in the seabed boundary layers. Therefore, an improved understanding of the fluid dynamics

Manuscript received: 12.08 .2004

Revised manuscript received: 18.03 .2005

Manuscript accepted: 20.03.2005 causing these situations of practical interest is essential to provide optimal solutions for the numerous requirements and problems associated with near-shore coastal areas.

The sea bottom in the near-shore areas is frequently covered with ripples. However, most laboratory measurements in combined wave and current flows have been carried out over flat beds (e.g., [1-5], among several others). These results are therefore directly relevant to plane seabeds because the near-bed flow structure above ripples could be different from that above a flat rough bottom owing to the organized periodic momentum transfer associated with ripples.

The limited number of experimental studies of combined wave-current motion over ripples include those reported in [6-8]. However, wave motion at right angles to a steady current was considered in [7] whilst the artificial vortex ripples used in [8] had sharp crests which are known to induce stronger separation vortices than naturally formed ripples with rounded crests. Consequently, the present study focuses on collinear wave and current flows close to a rippled bed whose profile is 
similar to that of naturally forming vortex ripples.

The primary objectives of the present paper are to examine, a) the effect of adding a collinear current on the ensemble velocity field of a wave, and $b$ ) the effect of introducing a wave on the time-mean velocities of a collinear current, in combined wave and current flow close to a bed of ripples.

\section{Experimental Set-up}

The experiments were carried out in an oscillatory flow water tunnel with facility for a re-circulating steady flow. This tunnel consists essentially of a U-tube in which water is caused to oscillate by a paddle driven by a variable speed motor. The rectangular working section of the tunnel is horizontal and $3.7 \mathrm{~m}$ long, $0.31 \mathrm{~m}$ wide and $0.45 \mathrm{~m}$ high. Under normal conditions without the steady current, the flow is almost sinusoidal, with the amplitude of the higher harmonics being less than $3 \%$ of the amplitude of the fundamental.

A rippled bed made out of hardwood was laid on the full length of the horizontal working section of the tunnel. The profile of the rippled bed used in the present study (see Fig. 1) is very similar to that of the ripples which form with $0.4 \mathrm{~mm}$ median diameter sand.

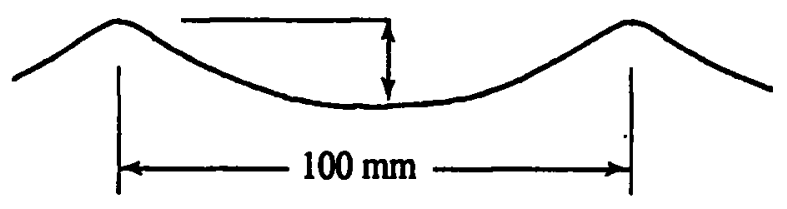

Figure 1: Profile of the rippled bed.

The fluid velocities, both the horizontal and the vertical components, denoted by $u$ and $v$, respectively, were measured simultaneously using a laser Doppler anemometer (LDA) in forward scatter mode. These measurements were made, first above the crest, and then at five other equally spaced sections upstream of the crest. The spacing between the sections was one-sixth of the ripple wave length.

In the steady flow alone tests, the two velocity components were sampled at a frequency of $0.05 \mathrm{kHz}$ over 100 seconds, after which the sampling programme returned the respective mean velocities and the root-mean-square fluctuations. On the other hand, the velocity was sampled 100 times per cycle for 50 cycles for the waves alone and combined wavecurrent tests. Ensemble- or phase-averaged (denoted in the following by e.g., $\bar{u}$ for the horizontal velocity component), mean- or time-averaged (e.g., $\bar{u}$ ) and root-mean-square velocities (e.g., $u_{\text {mms }}^{\prime}$ ) as well as these quantities averaged over a ripple length (denoted by square brackets) were then determined.

\section{Relevant Dimensionless Groups}

The independent variables representing the present fluid flow problem are $a$, the orbital amplitude of the fluid just outside the boundary layer; $h$, the ripple height; $L$, the ripple wave length; $t$, time; $T$, the period of oscillation; $\bar{u}_{{ }_{*} c}$, the shear velocity for the steady current; $x$, the distance measured in the horizontal direction from the ripple crest; $y$, the distance measured in the vertical direction from the bed and $v$, the kinematic viscosity of the fluid.

Thus, any dependent variable may be expressed as a function of :

$\frac{a}{L}, \frac{\beta}{k}, \frac{\overline{\bar{u}}_{w w c}}{U_{0}}, \frac{h}{L}, \frac{x}{L}, k y, \omega t$

where, $\omega=2 \pi / T, \beta=(\omega / 2 v) 1 / 2, k=2 \pi / L$ and $U_{0}$ is the amplitude of the free-stream velocity outside the boundary layer. Also note that the steady flow shear velocity $\vec{u}_{{ }}$, has been replaced by the shear velocity $\tilde{u}_{{ }_{\text {wc }}}$ relevant to the time-mean combined flow velocity. 


\section{Test Conditions}

The test conditions are summarized in Table 1.

Table 1: Test Conditions.

\begin{tabular}{|c|l|l|l|l|l|}
\hline $\begin{array}{c}\text { Test } \\
\text { no. }\end{array}$ & $\begin{array}{c}\text { Flow } \\
\text { combn. }\end{array}$ & $\begin{array}{c}U_{\text {o }} \\
(\mathrm{m} / \mathrm{s})\end{array}$ & $\begin{array}{c}T \\
(\mathrm{sec})\end{array}$ & $\frac{a}{L}$ & $\frac{\beta}{k}$ \\
\hline 1 & CL & $0.09 *$ & - & - & - \\
2 & $\mathrm{CS}$ & $0.06 *$ & - & - & - \\
\hline 3 & W1 & 0.12 & 4.53 & 0.84 & 14.3 \\
4 & W1CL & 0.11 & 4.53 & 0.82 & 14.8 \\
5 & W1CS & 0.11 & 4.56 & 0.80 & 14.6 \\
\hline 6 & W2 & 0.24 & 4.58 & 1.78 & 13.9 \\
7 & W2CL & 0.24 & 4.56 & 1.76 & 14.2 \\
8 & W2CS & 0.25 & 4.56 & 1.78 & 14.6 \\
\hline 9 & W3 & 0.44 & 4.51 & 3.18 & 14.3 \\
10 & W3CL & 0.44 & 4.58 & 3.20 & 14.0 \\
\hline
\end{tabular}

* Velocity at $y=100 \mathrm{~mm}$.

These experiments include measurements in combined wave-current flows as well as those made in the respective wave and current components separately. The combined flows investigated in the present study are wave dominant, and in such sea conditions, the values of $a / L$ and $h / L$ for ripples mostly fall between $0.4-10$ and $0.05-0.2$, respectively [9].

\section{Effect of Currents on Waves}

One objective of the present study is to examine the effect of adding a steady current on the phase-averaged velocity field of waves. Accordingly, Fig. 2 shows an example of the ensemble-mean velocity distribution at various phases over a cycle of oscillation, in the form of velocity vectors.
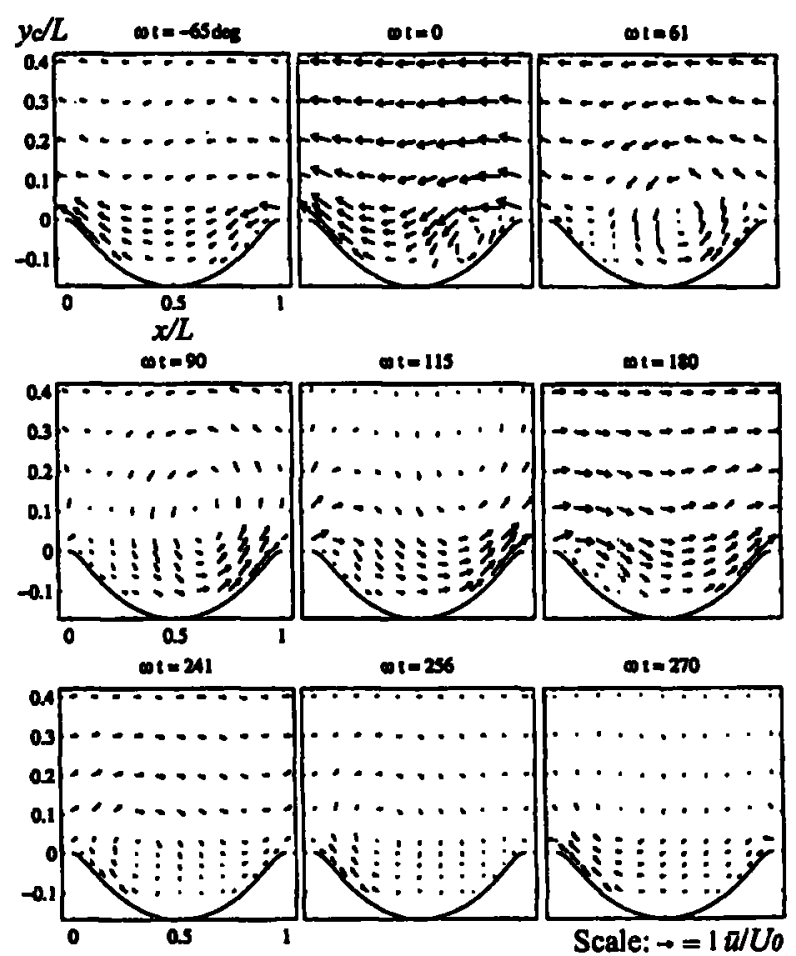

Figure 2: Velocity vectors in combined flow: Test no. 4.

Here, the vertical distances, $y_{c}$, are measured from the crest level of the ripples. Also, note that phase is measured relative to the maximum negative velocity in the free-stream and that the superimposed steady flow is from the right to the left of these figures. Accordingly, the wave motion is in the same direction as the superimposed steady flow during the half-cycle $-90^{\circ}<\omega t<+90^{\circ}$ and is in the opposite direction during $90^{\circ}<\omega t<270^{\circ}$

Fig. 2 clearly indicates that the lee-vortex formed during the half cycle $90^{\circ}<\omega t<270^{\circ}$ is considerably weaker' than that during $-90^{\circ}<\omega t<+90^{\circ}$. We also note that at the flow reversal, the stronger vortex is thrown out in a direction opposite to that of the superimposed steady flow whereas the weaker vortex is in the same direction. This asymmetry is expected to be significant because both the velocity and the turbulence fields above ripples are highly influenced by the dynamics of the lee vortices. Further, if the bed was movable we would expect asymmetric ripples under these conditions. 
Now, to examine the influence of the asymmetry in vorticity dynamics on the velocity field, let us compare the near-bed time variation of the phase-averaged horizontal component of velocity $\bar{u}$ with and without the superimposed steady current.

Accordingly, Fig. 3 shows the phase variation of $\bar{u}:$ a) above the crest, and b) above the trough.

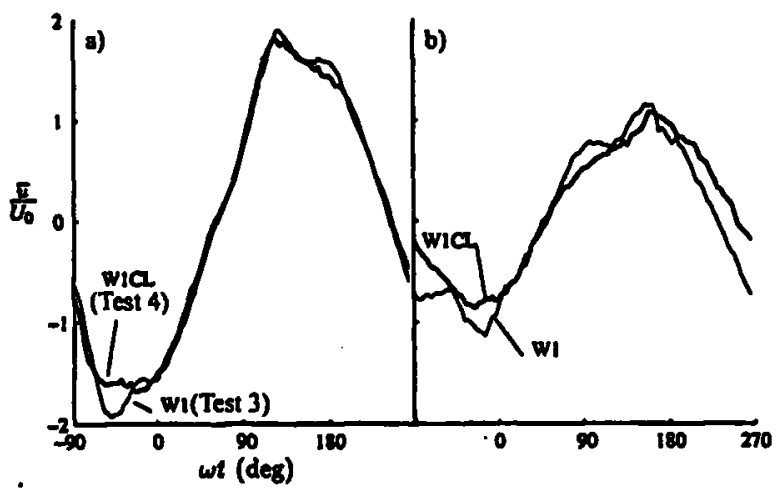

Figure 3: Examples of variation during the course of cycle of oscillation of $\bar{u}:(a) y_{c}=2 \mathrm{~mm}$ above crest, (b) $y_{c}=1 \mathrm{~mm}$ above trough.

At first sight, it appears that the addition of a steady current does not cause a significant influence on the near-bed velocity field. However, one notable feature in the combined flow (W1CL) time series above the crest is the weakening of the velocity peak at about $\omega t=-50^{\circ}$. The peak in the waves alone test (W1) is mainly due to the surge in the velocity associated with the ejection of the vortex formed during the previous half cycle. Therefore, it is not surprising that this peak is weaker in the combined flow test because the lee-vortex concerned is weaker, as mentioned earlier.

Similarly, the prominent peak at $\omega t \approx-20^{\circ}$ in the average velocity cycle above the trough for the waves alone test is weaker in the corresponding combined flow test. Further insight into the fluid dynamics associated with this may be obtained by examining the vorticity fields for these two tests at $\omega t \approx-20^{\circ}$ shown in Fig. 4. These plots reveal that the growing lee-vortex in the waves alone test shown in Fig. 4(b) has stretched over into the trough with the added impetus given by the passage of the previous half cycle vortex from the adjacent crest. On the other hand, the vorticity field for the corresponding combined flow test shown in Fig. 4(a) indicates that the previous half cycle vortex which is relatively weaker, is still beginning to emerge over the crest. Consequently, the growing lee-vortex in the combined flow test is not enhanced as significantly as in the former case, thus yielding lower velocities at $y_{c}=1 \mathrm{~mm}$ above the trough than in the respective waves alone test.

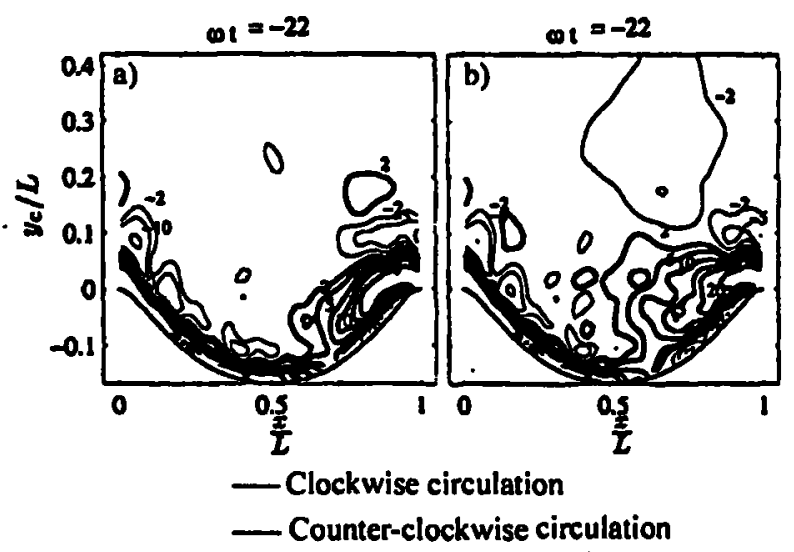

Figure 4: Vorticity contours at $\omega t \approx-22^{\circ}$ : (a) waves and currents, Test no. 4; (b) waves alone, Test no. 3.

Further, Fig. 5(a) compares the variation with height of the amplitude of the periodic component of the horizontal velocity above the crest in combined flow Test no. 4 and Test no. 5 with the same in the respective wave component alone, i.e., Test no. 3. The corresponding profiles above the trough are shown in Fig. 5(b).

The profiles, both above the crest and the trough, suggest an attenuation of the amplitude of the periodic component with the addition of the steady flow. This is mainly because the velocity peaks over ripples are largely determired by the dynamics of the leevortices, and for the two combined flow tests, the lee-vortex that grows against the steady 
flow is weaker resulting in smaller peaks over one half cycle.

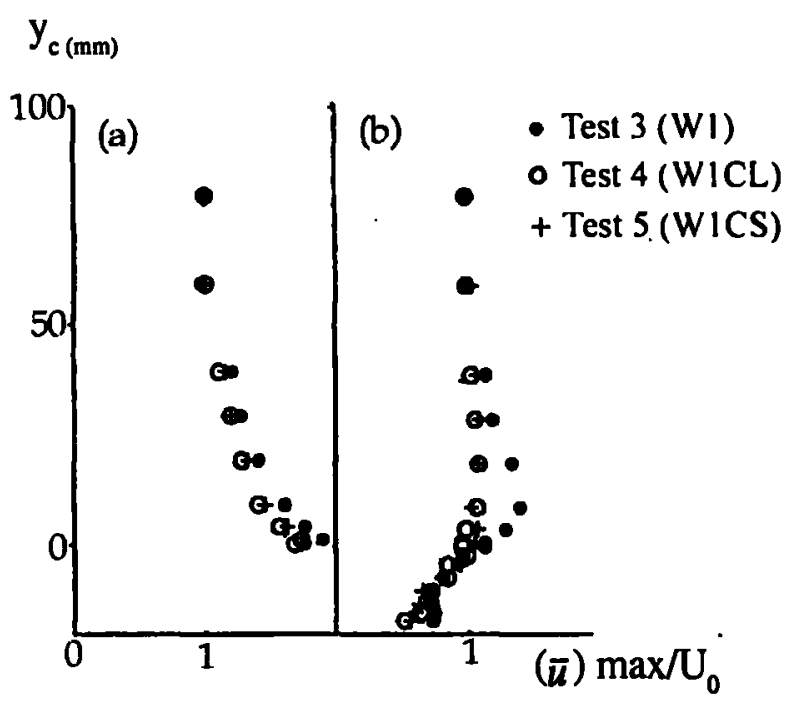

Figure 5: Variation with height of amplitude of the periodic component of horizontal velocity: (a) above crest, (b) above trough.

\section{Effect of Waves on Currents}

Time-mean velocities and bed shear in combined wave-current flows are responsible for most of the near-shore sediment and pollutant transport. Therefore, in the following, we examine the effect of introducing waves on the time-mean velocities and shear stresses of a steady current.

The way in which the time-averaged velocity field for the larger current (CL) is modified upon the addition of waves (W1) is shown in Fig. 6. The velocity field obtained for the smaller current (CS) is qualitatively similar to that of the larger current, and therefore, is not shown here. We see that the near-bed velocities in the region below the crest level have been strengthened whilst those above have been reduced considerably in comparison with those for the steady current alone. A similar reduction in the time-mean velocities has been reported in collinear combined flows over two- dimensional roughness [1] and above rippled beds with wave motion at right angles to the steady flow [7].

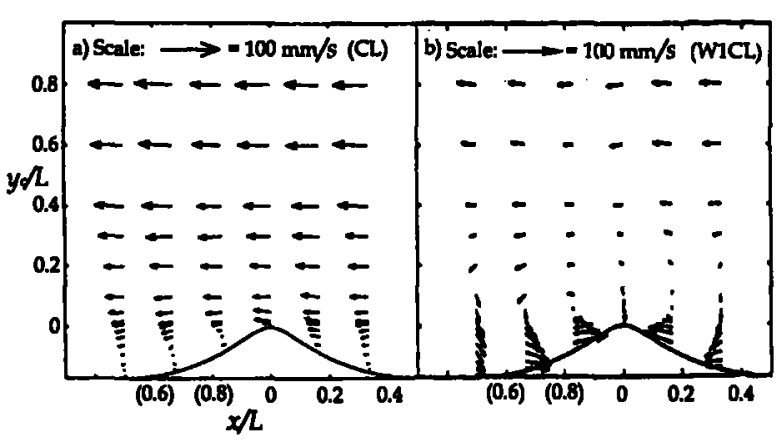

Figure 6: Time-mean velocity distributions: (a) steady flow alone, Test no. $1 ;(b)$ combined wave and current flow: Test no. 4.

One main consequence of superimposing a wave on the steady current is that the current in the region above the wave dominated layer experiences a shear stress, which depends not only on the physical bottom roughness but also on the wave boundary layer characteristics [10]. The shear velocity and the associated roughness experienced by the steady current, in isolation or in combination with a wave, is usually deduced from the gradient and the zero velocity intercept, respectively, of a semilogarithmic plot of velocity variation with height. Such plots of the spatially averaged time-mean velocities $([\overline{\bar{u}}])$ for the larger current (CL) are shown in Fig. 7, together with those in the same current combined with three different waves. The corresponding profiles for the smaller current (CS), and that combined with two different waves, are given in Fig. 8.

Here, we use spatially averaged velocities to minimise the effects of the wavy bottom on the time-mean profiless, particularly in the near-bed region. The origin for these profiles is taken as the mid-height between the ripple crest and the trough.

We shall first consider the steady flow alone velocity profiles. The velocity distribution appears to be logarithmic from $y=8.5 \mathrm{~mm}$, i.e., crest level, up to a height of about $50 \mathrm{~mm}$ for both CL and CS. The values of the shear velocity $\left(\overline{\bar{u}}_{t_{c}}\right)$ and the intercept $\left(\mathrm{y}_{0}\right)$ obtained by fitting straight lines to the logarithmic part of the velocity profile using standard regression 
techniques are given in Table 2. The corresponding correlation coefficients $\left(\mathrm{r}^{2}\right)$ and the $95 \%$ confidence interval for $\overline{\bar{u}}_{*_{c}}$ are also given.

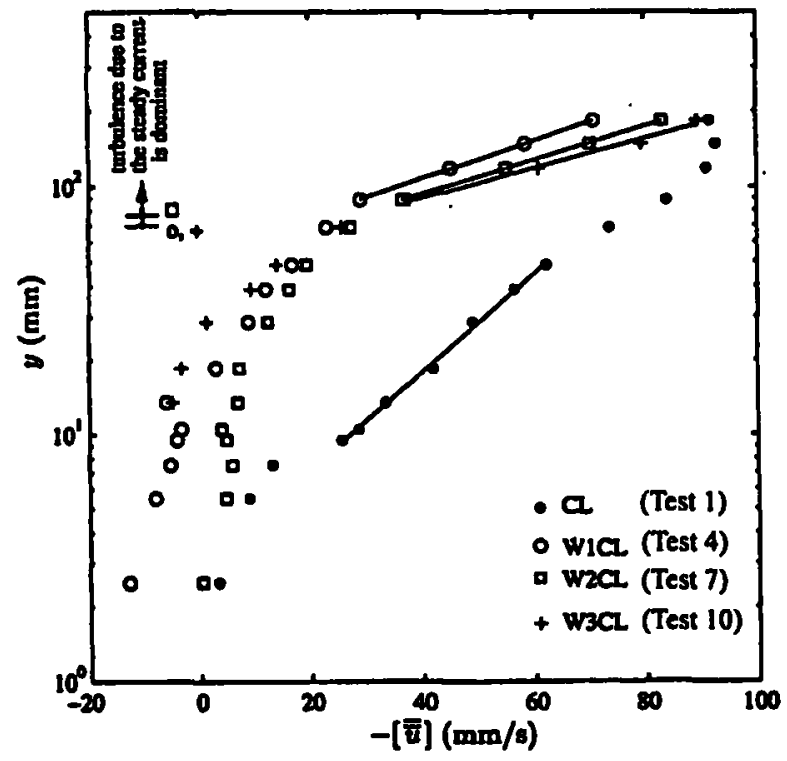

Figure 7: Spatially averaged time-mean velocities: waves combined with larger current.

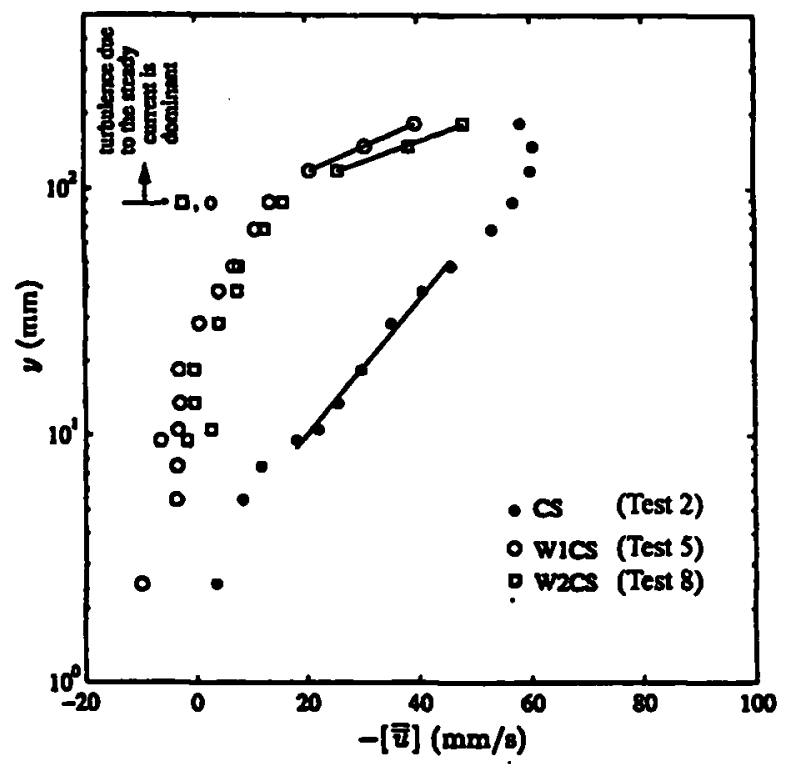

Figure 8: Spatially averaged time-mean velocities: waves combined with smaller current.
Table 2: Values of shear velocity and apparent roughness obtained by fitting semi-logarithmic lines to the measured time-mean velocity profiles.

\begin{tabular}{|c|l|l|l|l|l|}
\hline $\begin{array}{c}\text { Test } \\
\text { no. }\end{array}$ & $\begin{array}{c}\text { Flow } \\
\text { Combn }\end{array}$ & $\begin{array}{c}\overline{\bar{u}}_{{ }_{\psi_{r}}} \\
(\mathrm{~mm} / \mathrm{s})\end{array}$ & $\begin{array}{c}\mathrm{y}_{0}, \mathrm{y}_{\mathrm{q}} \\
(\mathrm{mm})\end{array}$ & $\mathbf{r}^{2}$ & $\begin{array}{c}95 \% \\
\text { confi- } \\
\text { dence } \\
\text { interval } \\
(\%)\end{array}$ \\
\hline 1 & $\mathrm{CL}$ & 9.0 & 2.88 & 0.9970 & \pm 4.9 \\
2 & $\mathrm{CS}$ & 6.4 & 2.75 & 0.9888 & \pm 9.6 \\
4 & W1CL & 23.4 & 53.37 & 0.9996 & \pm 4.1 \\
5 & W1CS & 17.6 & 72.76 & 0.9998 & \pm 8.9 \\
7 & W2CL & 26.3 & 49.92 & 0.9998 & \pm 2.9 \\
8 & W2CS & 20.9 & 70.96 & 0.9968 & \pm 35.7 \\
10 & W3CL & 29.9 & 51.84 & 0.9898 & \pm 20.9 \\
\hline
\end{tabular}

Now, a word of caution before we move on to the combined flow time-mean velocity profiles. The supposition of measured velocity profiles as logarithmic to obtain shear stress and roughness estimates must be done with great care: for example a slight curvature in the profile, perhaps due to secondary effects (e.g., density stratification, acceleration) may easily be disregarded leading to erroneous values of $u_{\text {. and }} y_{0}$ [11]. This is particularly relevant to the time-mean velocities in a combined flow situation where the mean velocity profile is likely to be perturbed by many factors: for instance over ripples, the near-bed time-mean flow is likely to be affected by the dynamics of the lee-vortices and the turbulence of the wave motion. Consequently, as a first step, it is vital to identify the region in which the logarithmic distribution is expected, from theoretical or practical considerations.

Accordingly, we utilize measured turbulence intensities to identify the semi-log regions in the combined flow velocity profiles. Two examples of the cycle-mean value of the spatially averaged turbulent fluctuations in the vertical component of velocity in combined wave-current flows and in the respective flow components separately are shown in Fig. 9. 


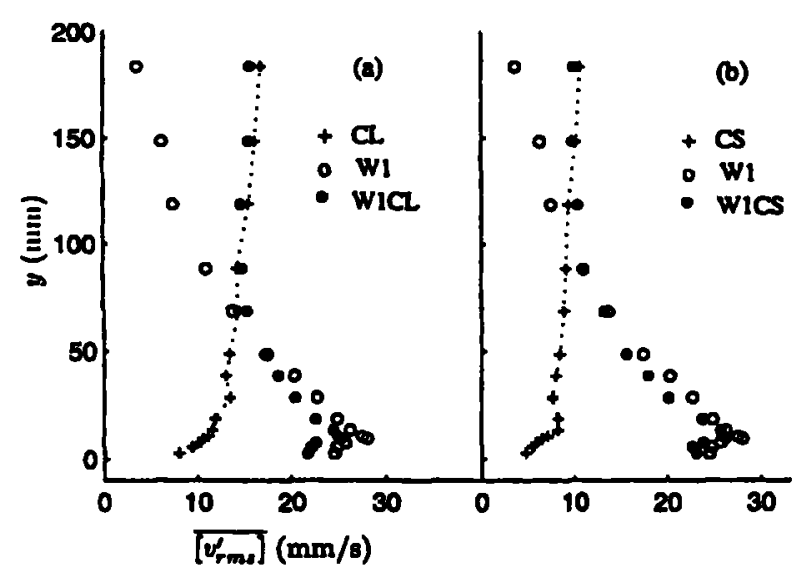

Figure 9: Variation with height of cycle-mean values of $/ v^{\prime}$ J a) larger current CL (Test no. 1), wave WI (Test no. 3), corresponding combined flow WICL (Test no. 4); b) smaller current CS (Test no. 2), wave WI (Test no. 3), corresponding combined flow WICS (Test no. 5).

The turbulence profiles in Fig. 9 show that an upper layer exists in which the cycle-mean value of the combined flow turbulence is dominated by the turbulence from the steady current. It also appears that the extent of this region is determined mainly by the relative strengths of the turbulence intensities in the pure component flows. Accordingly, this region is thicker for Test no. 4 where the larger current is combined with the wave W1 (Fig. 9(a)) than the same wave combined with the smaller current, Test no. 5 (Fig. 9(b)). Now, one would expect the semi-logarithmic layer to exist in the region where the combined flow turbulence is dominated by the turbulence from the steady flow component. We see that for Test no. 4 and Test no. 5, the turbulence due to the steady current is dominant for values of $y$ greater than about $70 \mathrm{~mm}$ and $90 \mathrm{~mm}$, respectively. Consequently, we would expect the semi-log layer to exist, for example, above $70 \mathrm{~mm}$ for Test no. 4. Similarly, the heights above which the turbulence due to the steady current is dominant, and so the semi-log layer to exist, were obtained for all combined flow tests and are indicated in Fig. 7 and Fig. 8.

We see that the combined flow time-mean velocity profiles in Fig. 7 and Fig. 8 show a relatively thin 'transition' region immediately above these heights, followed by a region in which the velocity profiles appear to be semilogarithmic. Unfortunately, measurements are not available for $y \geq 185 \mathrm{~mm}$ due to the limitations of the traversing range of the LDA. The straight lines drawn through the data points in the semi-logarithmic region were fitted using the same method as for the steady flow alone profiles. The values of the shear velocity corresponding to the time-mean part of the combined flow velocities in the horizontal direction $\left(\overline{\bar{u}}_{*_{w c}}\right)$ and the apparent roughness $\left(y_{a}\right)$ cobtained in this way are given in Table 2 together with the correlation coefficients $\left(r^{2}\right)$ and the $95 \%$ confidence intervals on $\overline{\bar{u}}_{*_{w c}}$. The $95 \%$ confidence band on $\overline{\bar{u}}_{\text {*wc }}$ for Test no. 8 is uniacceptably large despite the relatively high value of $r^{2}$. This is mainly because only three data points are available in the semi-logarithmic region for the regression analysis.

The time-mean velocity profiles inside the wave dominated region, above the crest level, appear to be slightly curved upwards. This, perhaps, reflects the fact that the roughness felt by the time-mean flow inside the wave dominated region is gradually increasing with height, as for instance suggested by Davies et al. [12]. It should, however, be added that the curved nature of the velocity profiles is not very clear in the wave dominated region of Test no. 5.

Clearly, an increase in the shear velocity experienced by the current outside the wave dominated region is evident, with the superimposition of wave motion (see column 3 in Table 2). It is also evident that the shear velocities gradually increase with the increasing wave strength, for the present combined flow tests. The values of the apparent roughness $\left(y_{a}\right)$ too show a considerable increase with the addition of the wave motion. Accordingly, these results further confirm that a combined wave-current flow is more efficient in eroding bottom sediment than current alone due to the increased bed shear stress. 
Moreover, it is interesting to note that the smaller current combined with a given wave motion yields a larger $y_{a}$ than that associated with the larger current combined with the same wave. This, however, is not surprising because the region of the flow affected by the wave generated turbulence is thicker for the smaller current than for the larger current, as we discussed earlier. In other words $y_{a} / y_{0}$, is dependent upon the relative wave-current strength $\left(u_{*_{w}}\right)_{\max } / u_{* c}$, with higher values of $\left(u_{*_{w}}\right)_{\max } / u_{*_{c}}$ yielding larger values of $y_{a}$ at a given a/L. It must, however, be added that the present experiments have been performed over a bed of fixed ripples, the flow resistance of which might be different to that over a bed of movable ripples.

\section{Conclusions}

Detailed measurements of the velocities in combined wave and current boundary layer flow over a fixed bed of vortex ripples $(h / L=$ 0.17 ) have been presented. The measured velocities have been analysed to examine the effect of adding a collinear current on the ensemble velocity field of a wave as well as the effect of the wave on the time-mean velocities of the current. For the range of conditions covered in these experiments the main conclusions are as follows:

1. In collinear combined flows, depending on the relative strength of the wave and current components, the lee-vortices that are formed during one half-cycle are weaker than those formed during the other half-cycle. Present measurements indicate that this asymmetry causes a significant effect on the velocity field in the near-bed region.

2. The velocity records, both above the crest and the trough, suggest an attenuation of the amplitude of the periodic component of the horizontal velocity with the addition of a steady flow.
3. The results also indicate that the steady flow time-mean velocities in the region below the crest level are strengthened whilst those above are reduced considerably with the addition of waves.

4. The values of shear velocity $\overline{\bar{u}}_{\text {.wc }}$ and apparent roughness $y_{a}$ deduced from logarithmic parts of the time-mean velocity profiles for combined flows are considerably larger than those for the steady component alone: increase in $\vec{u}$. and $y_{a}$ is by a factor of about $2.6-3.3$ and $18-29$, respectively.

\section{References}

1. W.T. Bakker and T. Van Doorn, "Nearbottom velocities in waves with a current", Proc. 16th Conf. Coastal Eng., Hamburg, pp. 1394-1413, 1978.

2. P.H. Kemp and R.R. Simons, "The interaction between waves and a turbulent current: waves propagating with the current", J. Fluid Mech., 116, pp. 227250, 1982.

3. J.F.A. Sleath, "Velocities and bed friction in combined flows", Proc. 22nd Conf. Coastal Eng., Delft, pp. 450-463, 1990.

4. R.R. Simons, T.J. Grass and M.M. Tehrani, "Bottom shear stresses in the boundary layers under waves and currents crossing at right angles", Proc. 23rd Conf. Coastal Eng., Venice, pp. 604-617, 1992.

5. C.R Lodhal, B.M. Sumer and J. Fredsoe, "Turbulent combined oscillatory and current in a pipe", J. Fluid Mech., 373, pp. 313-348, 1998.

6. I. Brevik and B. Aas, "Flume experiments on waves and currents 1: rippled bed", Coastal Eng., 3, pp. 149-177, 1980.

7. K. I. M. Ranasoma and J. F. A. Sleath, "Combined oscillatory and steady flow over ripples", ASCE J. Waterway, Port, 
Coastal and Ocean Eng. Div., 120 (4), pp. 331-346, 1994.

8. J. Fredsoe, K.H. Andersen and M. Sumer, "Wave plus current over a ripple covered bed", Coastal Eng., 38, pp. 177-221, 1999.

9. P. Nielsen, Coastal Bottom Boundary Layers and Sediment Transport, Singapore: World Scientific, 1992.

10. W.D. Grant and O.S. Madsen, "Combined wave and current interaction with a rough bottom", J. Geophys. Res., 84 (C4), pp. 1797-1808, 1979.

11. R.L. Soulsby, "The bottom boundary layer of shelf seas", pp. 189-266, in B. Johns, Physical Oceanography of Coastal and Shelf Seas, Amsterdam: Elsvier, 1983.
12. A.G. Davies, R.L. Soulsby and H.L. King, "A numerical model of the combined wave and current bottom boundary layer", $J$. Geophys. Res., 93 (Cl), pp. 491-508, 1988.

\section{Biographical Sketch}

JANAKA J. WIJETUNGE, B.Sc. Eng. Hons., Ph.D. (Cambridge), AMIE (SL). Dr Wijetunge is a Senior Lecturer in Civil Engineering at the Department of Civil Engineering, University of Peradeniya. He has specialised in Coastal Hydraulics at Cambridge University and his current research interests include wave-current and wave-structure interactions in shallow coastal waters. 